\title{
ON THE RATIONAL COHOMOLOGY OF THE TOTAL SPACE OF THE UNIVERSAL FIBRATION WITH AN ELLIPTIC FIBRE
}

\author{
KATSUHIKO KURIBAYASHI
}

\begin{abstract}
Let $\mathcal{F}_{M}$ be the universal fibration having fibre $M$ an elliptic space with vanishing odd rational cohomology. We consider the rational cohomology of the total space of $\mathcal{F}_{M}$ by using a function space model due to Haefliger, Brown and Szczarba when Halperin's conjecture is affirmatively solved for the fibre. The calculation enables one to deduce that the cohomology of the classifying space of the self-homotopy equivalences of a c-symplectic space $M$ is generated only by the Kedra-McDuff $\mu$-classes if the cohomology of $M$ is generated by a single element.
\end{abstract}

\section{INTRODUCTION}

Let $M$ be an elliptic space, namely a simply-connected space whose rational homotopy and cohomology are finite-dimensional. Suppose further that the Euler characteristic $\sum_{i}(-1)^{i} \operatorname{dim} H^{*}(M ; \mathbb{Q})$ is positive. Following Lupton [19], we call such a space positively elliptic. Then Halperin's conjecture states that for any positively elliptic space $M$, each fibration $M \rightarrow E \stackrel{p}{\rightarrow} B$ with a simply-connected base is TNCZ; that is, the Leray-Serre spectral sequence with coefficients in the rational field for the fibration collapses at the $E_{2}$-term. In this case the cohomology $H^{*}(E ; \mathbb{Q})$ is isomorphic to the tensor product $H^{*}(B ; \mathbb{Q}) \otimes H^{*}(M ; \mathbb{Q})$ as an $H^{*}(B ; \mathbb{Q})$-module, where the module structure is defined by the induced map $p^{*}: H^{*}(B ; \mathbb{Q}) \rightarrow H^{*}(E ; \mathbb{Q})$. We observe that, as an algebra, $H^{*}(E ; \mathbb{Q})$ is not isomorphic in general to the tensor product with the natural algebraic structure induced by the products on the factors.

We mention that the conjecture has been affirmatively solved in some cases; see [35], [27], [31], [20] and [22]. Our interest here lies in investigating the algebra structure of the rational cohomology of the total space of a fibration with a fibre for which Halperin's conjecture is affirmatively solved.

In the rest of this section, we describe our results. Let aut ${ }_{1}(M)$ be the component of the monoid of the self-homotopy equivalences of a space $M$ containing the identity and $M \stackrel{\iota}{\rightarrow} M_{\text {aut }_{1}(M)} \stackrel{\pi}{\rightarrow} B_{a_{1}}(M)$ the universal $M$-fibration [24]. Suppose that $M$ is a simply-connected space whose rational cohomology is isomorphic to an algebra of the form

$$
\mathbb{Q}\left[x_{1}, \ldots, x_{q}\right] /\left(u_{1}, \ldots, u_{q}\right),
$$

2000 Mathematics Subject Classification: 55P62, 57R19, 57R20, 57T35.

Key words and phrases. Self-homotopy equivalence, c-symplectic manifold, homogeneous space, Sullivan model, evaluation map, characteristic class, the Eilenberg-Moore spectral sequence.

This research was partially supported by a Grant-in-Aid for Scientific Research (C)20540070 from Japan Society for the Promotion of Science.

Department of Mathematical Sciences, Faculty of Science, Shinshu University, Matsumoto, Nagano 390-8621, Japan e-mail:kuri@math.shinshu-u.ac.jp 
where $\left\{u_{1}, \ldots, u_{q}\right\}$ is a regular sequence. Observe that the cohomology of every positively elliptic space is of the form (1.1) for some $q$ and conversely, a simply connected space whose cohomology has the form (1.1) is positively elliptic; see [9] and [4].

In what follows, for an augmented algebra $A$, we denote by $A^{+}$the augmentation ideal. Let $\left\{m_{j}\right\}_{j \in J}$ be an arbitrary basis of the cohomology $H^{*}(M ; \mathbb{Q})$ with $J=$ $J^{\prime} \coprod\{0\}$ and $m_{0}=1$. By using a minimal model for $M$ and the basis $\left\{m_{j}\right\}_{j \in J}$, we can construct a model for $\operatorname{aut}_{1}(M)$ due to Haefliger [8], Brown and Szczarba [1], which is called the HBS model. Let $e v: \operatorname{aut}_{1}(M) \times M \rightarrow M$ be the evaluation map defined by $e v(\gamma, x)=\gamma(x)$ for $\gamma \in \operatorname{aut}_{1}(M)$ and $x \in M$. Then an explicit model for the evaluation map is constructed with the HBS model for $\operatorname{aut}_{1}(M)$; see [2], [16] and [17]. Such the models enable one to determine $H^{*}\left(\operatorname{Baut}_{1}(M) ; \mathbb{Q}\right)$-linear parts of the relations in the algebra $H^{*}\left(M_{\mathrm{aut}_{1}(M)} ; \mathbb{Q}\right)$. More precisely we establish

Theorem 1.1. Let $M$ be a simply-connected space whose rational cohomology is of the form (1.1). Suppose that all derivations of negative degree of $H^{*}(M ; \mathbb{Q})$ vanish. Then there exist a subset $S$ of the set $\{1, \ldots, q\} \times J$ containing $S_{0}:=\{(i, 0) \mid 1 \leq i \leq q\}$ and indecomposable elements $\nu_{s}$ of $H^{*}\left(\operatorname{Baut}_{1}(M) ; \mathbb{Q}\right)$ indexed by $S$ such that

$$
H^{*}\left(\operatorname{Baut}_{1}(M) ; \mathbb{Q}\right) \cong \mathbb{Q}\left[\nu_{s} \mid s \in S\right]
$$

as an algebra and

$$
H^{*}\left(M_{\text {aut }_{1}(M)} ; \mathbb{Q}\right) \cong \mathbb{Q}\left[\nu_{s} \mid s \in S\right] \otimes \mathbb{Q}\left[x_{1}, \ldots, x_{q}\right] /\left(u_{i}-\sum_{s=(i, j) \in S} \nu_{s} m_{j}+D_{i}\right)
$$

as an $H^{*}\left(\operatorname{Baut}_{1}(M) ; \mathbb{Q}\right)$-algebra, where each $D_{i}$ is an appropriate decomposable element in the ideal generated by $\pi^{*}\left(H^{*}\left(\operatorname{Baut}_{1}(M) ; \mathbb{Q}\right)^{+} \cdot H^{*}\left(\operatorname{Baut}_{1}(M) ; \mathbb{Q}\right)^{+}\right)$, $\iota^{*}\left(x_{i}\right)=x_{i}$ for $i=1, \ldots, q$ and $\operatorname{deg} \nu_{(i, j)}=\operatorname{deg} u_{i}-\operatorname{deg} m_{j}$ for $(i, j) \in S$. Moreover one has

$$
\begin{aligned}
\sharp\left\{\nu_{s} \mid \operatorname{deg} \nu_{s}=2 k, s \in S\right\}= & \operatorname{rank} \oplus_{a-b=2 k-1} \pi_{a}(M) \otimes H^{b}(X ; \mathbb{Q}) \\
& -\operatorname{rank} \oplus_{a-b=2 k} \pi_{a}(M) \otimes H^{b}(X ; \mathbb{Q}) .
\end{aligned}
$$
have

For any $s \in S_{0}$, the element $\nu_{s}$ is decomposable in $H^{*}\left(M_{\text {aut }_{1}(M)} ; \mathbb{Q}\right)$. Thus we

Corollary 1.2. As an algebra,

$$
H^{*}\left(M_{\text {aut }_{1}(M)} ; \mathbb{Q}\right) \cong \mathbb{Q}\left[\nu_{s} \mid s \in S-S_{0}\right] \otimes \mathbb{Q}\left[x_{1}, \ldots, x_{q}\right] .
$$

The derivations of $H^{*}(M ; \mathbb{Q})$ are closely related with the Halperin conjecture. Indeed, the result [27, Theorem A] due to Meier asserts that the conjecture is true for a positively elliptic fibre $M$ if and only if all derivations of negative degree of $H^{*}(M ; \mathbb{Q})$ vanish. Thus Theorem 1.1 is applicable to spaces satisfying Halperin's conjecture, for example, the homogeneous space $G / H$ for which $G$ is a Lie group and $H$ is a subgroup with $\operatorname{rank} G=\operatorname{rank} H$; see [31].

We mention that Lemma 3.1 below describes a necessary and sufficient condition for the vector space of negative derivations of $H^{*}(M ; \mathbb{Q})$ to be trivial in terms of the differential $\delta$ of the HBS model for $\operatorname{aut}_{1}(M)$. We also observe that the subset $S$ in Theorem 1.1 is determined when choosing a basis for the image of the linear part of $\delta$; see Section 3 for more details. 
We can arrange the given basis $\left\{m_{j}\right\}_{j \in J}$ of $H^{*}(M ; \mathbb{Q})$ so that $D_{i}=0$ for any $i$. This follows from deformation theory as we see in [10, Section 3]. It is mentioned that the suitable basis of $H^{*}(M ; \mathbb{Q})$ are chosen by considering the vector space of infinitesimal deformations $T_{\mathbb{Q}}^{1}\left(H^{*}(M ; \mathbb{Q})\right)$ of $H^{*}(M ; \mathbb{Q})$. By virtue of $[10$, Theorem $3.1]$, the cohomology ring $H^{*}\left(M_{\text {aut }_{1}(M)} ; \mathbb{Q}\right)$ is viewed as an equivariant versal deformation of $H^{*}(M ; \mathbb{Q})$ along the ring $H^{*}\left(\operatorname{Baut}_{1}(M) ; \mathbb{Q}\right)$. Moreover a formula of the rational homotopy group of $M_{\mathrm{aut}_{1}(M)}$ is described in [10, Theorem D] in terms of the infinitesimal deformations. The proof of $[10$, Theorem D] then yields Corollary 1.2 .

The novelty here is that the generators $\nu_{s}$ of $H^{*}\left(\operatorname{Baut}_{1}(M) ; \mathbb{Q}\right)$ are related to those of the HBS model for $\operatorname{aut}_{1}(M)$ via the Eilenberg-Moore spectral sequence converging to $H^{*}\left(M_{\text {aut }_{1}(M)} ; \mathbb{Q}\right)$; see Section 5 . We also stress that our computation of $H^{*}\left(M_{\text {aut }_{1}(M)} ; \mathbb{Q}\right)$ is started with an arbitrary basis of $H^{*}(M ; \mathbb{Q})$. In addition, we obtain the algebra structure of $H^{*}\left(M_{\mathrm{aut}_{1}(M)} ; \mathbb{Q}\right)$ without depending on deformation theory.

We refer the reader to [5, Theorems 1, 2 and 3], [27, Proposition 1] and [33, Theorem 3.1] for the rational homotopy group of $\operatorname{aut}_{1}(M)$ for an elliptic space $M$ and a more general two-stage space.

Let $M \rightarrow E \rightarrow B$ be a fibration over a simply-connected space $B$. Assume that the cohomology of $M$ is of the form (1.1) and that all derivations of negative degree of $H^{*}(M ; \mathbb{Q})$ vanish. As mentioned in [27, (2.12)], the Eilenberg-Moore spectral sequence argument enables us to conclude that, as an $H^{*}(B ; \mathbb{Q})$-algebra,

$$
\begin{aligned}
H^{*}(E ; \mathbb{Q}) & \cong H^{*}(B ; \mathbb{Q}) \otimes_{H^{*}\left(B \operatorname{aut}_{1}(M) ; \mathbb{Q}\right)} H^{*}\left(M_{\text {aut }_{1}(M)} ; \mathbb{Q}\right) \\
& \cong H^{*}(B ; \mathbb{Q}) \otimes \mathbb{Q}\left[x_{1}, \ldots, x_{q}\right] /\left(u_{i}-\sum_{s=(i, j) \in S} f^{*}\left(\nu_{s}\right) m_{j}+f^{*}\left(D_{i}\right)\right),
\end{aligned}
$$

where $f: B \rightarrow$ Baut $_{1}(M)$ is the classifying map of the given fibration. In particular, one can obtain the algebra structure of $H^{*}(E ; \mathbb{Q})$ if $B$ is a suspension space because $f^{*}\left(D_{i}\right)=0$ for $i=1, \ldots, q$.

In a particular case, the $H^{*}\left(\operatorname{Baut}_{1}(M) ; \mathbb{Q}\right)$-algebra structure of $H^{*}\left(M_{\text {aut }}(M) ; \mathbb{Q}\right)$ is determined explicitly.

Proposition 1.3. Let $M$ be a simply-connected space such that $H^{*}(M ; \mathbb{Q}) \cong$ $\mathbb{Q}[a] /\left(a^{m+1}\right)$. Then

$$
H^{*}\left(\operatorname{Baut}_{1}(M) ; \mathbb{Q}\right) \cong \mathbb{Q}\left[\nu_{2}, \ldots, \nu_{m+1}\right]
$$

as an algebra and

$$
H^{*}\left(M_{\text {aut }_{1}(M)} ; \mathbb{Q}\right) \cong \mathbb{Q}\left[\nu_{2}, \ldots, \nu_{m+1}\right] \otimes \mathbb{Q}[a] /\left(a^{m+1}-\sum_{s=2}^{m+1} \nu_{s} a^{m-s+1}\right)
$$

as an $H^{*}\left(\operatorname{Baut}_{1}(M) ; \mathbb{Q}\right)$-algebra, where $\operatorname{deg} \nu_{k}=2 k$.

This result also follows from the computation in [6, Section 3] due to Gatsinzi.

The following subject is characterization of the indecomposable elements of $H^{*}\left(\operatorname{Baut}_{1}(M) ; \mathbb{Q}\right)$ mentioned in Theorem 1.1. Following Kedra and McDuff [13], we introduce characteristic classes of $\operatorname{Baut}_{1}(M)$ with fibre integration of the universal $M$-fibration. The calculation of $H^{*}\left(M_{\text {aut }_{1}(M)} ; \mathbb{Q}\right)$ in Theorem 1.1 allows us to conclude that the characteristic classes coincide with the indecomposable elements $\nu_{s}$ of $H^{*}\left(\operatorname{Baut}_{1}(M) ; \mathbb{Q}\right)$ modulo decomposable elements. To see this more 
precisely, we recall that $(M, a)$ is a cohomologically symplectic (c-symplectic) space with formal dimension $2 m$ if $M$ satisfies Poincaré duality over $\mathbb{Q}$ and $a$ is a class in $H^{2}(M ; \mathbb{Q})$ such that $a^{m} \neq 0$; see $[21]$.

Assume further that $M$ is a closed manifold and let $\mathcal{H}_{a}$ denote the group of diffeomorphisms of $M$ that fix $a$. Kedra and McDuff defined in [13, Section 3] cohomology classes, which are called the $\mu$-classes, of the classifying space $B \mathcal{H}_{a}$ provided $H^{1}(M ; \mathbb{Q})=0$. These classes are generalization of the characteristic classes of the classifying space of the group of Hamiltonian symplectomorphisms due to Reznikov [30] and Januszkiewicz and Kedra [12]. By the same way, for a symplectic space $(M, a)$ mentioned above, we can define characteristic classes $\mu_{k}$ of the classifying space $\operatorname{Baut}_{1}(M)$ with degree $2 k$ for $2 \leq k \leq m+1$. The class $\mu_{k}$ is also called the $k$ th $\mu$-class; see Section 6 for more details.

We can characterize the generators of $H^{*}\left(\operatorname{Baut}_{1}(M) ; \mathbb{Q}\right)$ in Proposition 1.3 by $\mu$-classes if $M$ is a c-symplectic space.

Proposition 1.4. Let $M$ be a simply-connected c-symplectic space whose rational cohomology $M$ is of the form $\mathbb{Q}[a] /\left(a^{m+1}\right)$. Then $H^{*}\left(\operatorname{Baut}_{1}(M) ; \mathbb{Q}\right)$ is generated by Kedra-McDuff $\mu$-classes:

$$
H^{*}\left(\operatorname{Baut}_{1}(M) ; \mathbb{Q}\right) \cong \mathbb{Q}\left[\mu_{2}, \mu_{3}, \ldots, \mu_{m+1}\right] .
$$

We give a computational example. Let $G$ be a Lie group, $K$ and $H$ subgroups of $G$. We define a map

$$
\lambda_{K, G / H}: K \rightarrow \operatorname{aut}_{1}(G / H)
$$

by $\lambda_{K, G / H}(g)(m)=g m$ with the left translation of $K$ on $G / H$, where $g \in K$ and $m \in G / H$. We observe that $\lambda_{K, G / H}$ is a monoid map and hence it induces the map $B \lambda_{K, G / H}: B K \rightarrow \operatorname{Baut}_{1}(G / H)$ between classifying spaces. Consider the real Grassmann manifold $M$ of the form $S O(2 m+1) / S O(2) \times S O(2 m-1)$. Since $H^{*}(M) \cong \mathbb{Q}[\chi] /\left(\chi^{2 m}\right)$ as an algebra, it follows from Proposition 1.4 that

$$
H^{*}\left(\operatorname{Baut}_{1}(M) ; \mathbb{Q}\right) \cong \mathbb{Q}\left[\mu_{2}, \mu_{3}, \ldots, \mu_{2 m}\right] .
$$

Observe that $\chi \in H^{2}(M ; \mathbb{Q})$ is the element which comes from the Euler class $\chi \in H^{2}(B S O(2) ; \mathbb{Q})$ via the map

$$
j^{*}: H^{*}\left(B(S O(2) \times S O(2 m-1) ; \mathbb{Q}) \cong \mathbb{Q}\left[\chi, p_{1}^{\prime}, \ldots, p_{m-1}^{\prime}\right] \rightarrow H^{*}(M ; \mathbb{Q}),\right.
$$

where $j$ is the fibre inclusion of the fibration

$$
M \stackrel{j}{\rightarrow} B(S O(2) \times S O(2 m-1)) \stackrel{B i}{\rightarrow} B S O(2 m+1) .
$$

Recall that the rational cohomology of $B S O(2 m+1)$ is a polynomial algebra generated by Pontrjagin classes; that is, $H^{*}(B S O(2 m+1) ; \mathbb{Q}) \cong \mathbb{Q}\left[p_{1}, \ldots, p_{m}\right]$, where $\operatorname{deg} p_{i}=4 i$. We relate the Pontrjagin classes to the $\mu$-classes with the map induced by $\lambda_{S O(2 m+1), M}$. More precisely, we have

Proposition 1.5. $\left(B \lambda_{S O(2 m+1), M}\right)^{*}\left(\mu_{2 i}\right) \equiv p_{i}$ modulo decomposable elements.

Let $\operatorname{Diff}_{1}(M)$ be the identity component of the group of diffeomorphisms of a manifold $M$ and $\left(\mathcal{H}_{a}\right)_{1}$ the subgroup of $\operatorname{Diff}_{1}(M)$ which fix the class $a$. Let Homeo $_{1}(M)$ denote the identity component of the group of homeomorphisms of $M$. The naturality of the integration along the fibre implies that the $k$ th KedraMcDuff $\mu$-classes of $B \operatorname{Diff}_{1}(M), B\left(\mathcal{H}_{a}\right)_{1}$ and of $B \operatorname{Homeo}_{1}(M)$ are extendable to the class $\mu_{k}$ in $H^{2 k}\left(\operatorname{Baut}_{1}(M) ; \mathbb{Q}\right)$. Thus an algebraic property of $\mu$-classes is deduced as a corollary to Proposition 1.5. 
Corollary 1.6. Let $M$ be the Grassmann manifold of the form $S O(2 m+1) / S O(2) \times$ $S O(2 m-1)$. Then the $\mu$-classes $\mu_{2}, \mu_{4}, \ldots, \mu_{2 m}$ in the cohomology $H^{*}\left(B \operatorname{Diff}_{1}(M) ; \mathbb{Q}\right)$, $H^{*}\left(B\left(\mathcal{H}_{a}\right)_{1} ; \mathbb{Q}\right)$ and $H^{*}\left(B \operatorname{Homeo}_{1}(M) ; \mathbb{Q}\right)$ are algebraically independent.

The proof of Proposition 1.5 also allows us to conclude that the image of the $k$ th $\mu$-class by the induced map

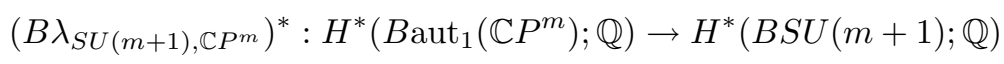

coincides with the $k$ th Chern class up to sign modulo decomposable elements; see the proof of [13, Proposition 1.7]. Observe that $\mathbb{C} P^{m} \cong U(m+1) / U(1) \times U(m)$. In order to prove Proposition 1.5, we will heavily rely on an explicit model for the left translation by a Lie group on a homogeneous space, which is investigated in [17, Sections 3 and 4]. This also illustrates usefulness of such an algebraic model.

The rest of this manuscript is set out as follows. In Section 2, we recall briefly the HBS model for $\operatorname{aut}_{1}(M)$ and a model for the evaluation map. A minimal model for the monoid is constructed in Section 3. Section 4 is devoted to constructing an approximation to the Eilenberg-Moore spectral sequence. In Section 5, we prove Theorem 1.1. After recalling $\mu$-classes, we prove Propositions 1.4 and 1.5 in Section 6.

\section{Models For the MONOID $\operatorname{aut}_{1}(M)$ AND FOR THE EVALUATION MAP}

In what follows, $H^{*}(-)$ and $H_{*}(-)$ denote the cohomology and homology with coefficients in the rational field, respectively.

Let $M$ be a formal space with $\operatorname{dim} H^{*}(M)<\infty$. We recall a Sullivan model for $\operatorname{aut}_{1}(M)$ and a model for the evaluation map ev : $\operatorname{aut}_{1}(M) \times M \rightarrow M$; see [8], [1], [5], [2] and [17] for more general function space models.

Let $\alpha:(\wedge V, d) \stackrel{\simeq}{\rightarrow} A_{P L}(M)$ be a minimal model for $M$, where $A_{P L}(M)$ denotes the commutative differential graded algebra of differential polynomial forms on $M$. Since $M$ is formal by assumption, there exists a quasi-isomorphism $\eta:(\wedge V, d) \stackrel{\simeq}{\rightrightarrows}$ $H^{*}(M)$.

Consider a differential graded algebra of the form $\left(\wedge\left(\wedge V \otimes B_{*}\right), D=d \otimes 1\right)$, where $B_{*}=H_{-*}(M)$. Let $\Delta$ be the coproduct of $H_{*}(M)$. We denote by $I$ the ideal of $\wedge\left(\wedge V \otimes H_{*}(M)\right)$ generated by $1 \otimes 1_{*}-1$ and all elements of the form

$$
a_{1} a_{2} \otimes \beta-\sum_{i}(-1)^{\left|a_{2}\right|\left|\beta_{i}^{\prime}\right|}\left(a_{1} \otimes \beta_{i}^{\prime}\right)\left(a_{2} \otimes \beta_{i}^{\prime \prime}\right),
$$

where $a_{1}, a_{2} \in \wedge V, \beta \in B_{*}$ and $\Delta(\beta)=\sum_{i} \beta_{i}^{\prime} \otimes \beta_{i}^{\prime \prime}$. The result [1, Theorem 3.5] implies that the composite $\rho: \wedge\left(V \otimes B_{*}\right) \hookrightarrow \wedge\left(\wedge V \otimes B_{*}\right) \rightarrow \wedge\left(\wedge V \otimes B_{*}\right) / I$ is an isomorphism of graded algebras. Moreover, it follows from [1, Theorem 3.3] that $D I \subset I$. Thus we have a DGA $(E, \delta)$ of the form

$$
E=\wedge\left(V \otimes B_{*}\right) \text { and } \delta=\rho^{-1} D \rho .
$$

Observe that, for elements $v \in V$ and $e \in B_{*}$, if $d(v)=v_{1} \cdots v_{m}$ with $v_{i} \in V$ and $D^{(m-1)}(e)=\sum_{j} e_{j_{1}} \otimes \cdots \otimes e_{j_{m}}$, then

$$
\delta(v \otimes e)=\sum_{j} \pm\left(v_{1} \otimes e_{j_{1}}\right) \cdots\left(v_{m} \otimes e_{j_{m}}\right) .
$$

Here the sign is determined by the Koszul rule; that is, $a b=(-1)^{\operatorname{deg} a \operatorname{deg} b} b a$ in a graded algebra. 
We define a DGA map $\left.u: \wedge\left(\wedge V \otimes B_{*}\right) / I\right) \rightarrow \mathbb{Q}$ by

$$
u(a \otimes b)=(-1)^{\tau(|a|)} b(\eta(a)),
$$

where $\tau(n)=[(n+1) / 2], a \in \wedge V$ and $b \in B_{*}$. Let $M_{u}$ be the ideal of $E$ generated by the set

$$
\{\omega \mid \operatorname{deg} \omega<0\} \cup\{\delta \omega \mid \operatorname{deg} \omega=0\} \cup\{\omega-u(\omega) \mid \operatorname{deg} \omega=0\} .
$$

Then the result [1, Theorem 6.1] asserts that $\left(E / M_{u}, \delta\right)$ is a model for $\operatorname{aut}_{1}(M)$. This means that there exists a quasi-isomorphism $\xi:\left(E / M_{u}, \delta\right) \rightarrow A_{P L}\left(\operatorname{aut}_{1}(M)\right)$. We call the DGA $\left(E / M_{u}, \delta\right)$ the HBS model for $\operatorname{aut}_{1}(M)$.

The proof of [16, Theorem 4.5] and [11, Remark 3.4] enable us to construct a model for the evaluation map ev : $\operatorname{aut}_{1}(M) \times M \rightarrow M$; see also [2]. The explicit form of the model is described in Proposition 2.1 below.

Let $\left\{m_{j}\right\}_{j \in J}$ be a basis of $H^{*}(M)$ and $\left\{\left(m_{j}\right)_{*}\right\}_{j \in J}$ the dual basis to $\left\{m_{j}\right\}_{j \in J}$. Then there exists a set $\left\{m_{j}^{\prime}\right\}_{j \in J}$ of linearly independent elements in $\wedge V$ such that $\eta\left(m_{j}^{\prime}\right)=m_{j}$.

Proposition 2.1. [17, Proposition 2.3, Remark 2.5(ii)] With the same notation as above, we define a map $m(e v):(\wedge V, d) \rightarrow\left(E / M_{u}, \delta\right) \otimes \wedge V$ by

$$
m(e v)(x)=\sum_{j}(-1)^{\tau\left(\left|m_{j}\right|\right)} \pi\left(x \otimes\left(m_{j}\right)_{*}\right) \otimes m_{j}^{\prime},
$$

for $x \in A$, where $\pi: E \rightarrow E / M_{u}$ denotes the natural projection. Then $m(e v)$ is a Sullivan representative for the evaluation map ev : $\operatorname{aut}_{1}(M) \times M \rightarrow M$; that is, there exists a homotopy commutative diagram

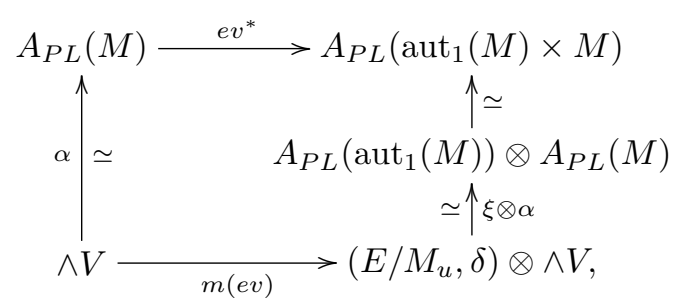

in which $\xi:\left(E / M_{u}, \delta\right) \stackrel{\simeq}{\rightarrow} A_{P L}\left(\operatorname{aut}_{1}(M)\right)$ is the Sullivan model for aut $_{1}(M)$ mentioned above.

Example 2.2. Let $M$ be a space whose rational cohomology is isomorphic to the truncated algebra $\mathbb{Q}[x] /\left(x^{m}\right)$, where $\operatorname{deg} x=l$. Recall the model $\left(E / M_{u}, \delta\right)$ for $\operatorname{aut}_{1}(M)$ mentioned in [11, Example 3.6]. Since the minimal model for $M$ has the form $(\wedge(x, y), d)$ with $d y=x^{m}$, it follows that

$$
E / M_{u}=\wedge\left(x \otimes 1_{*}, y \otimes\left(x^{s}\right)_{*} ; 0 \leq s \leq m-1\right)
$$

with $\delta\left(x \otimes 1_{*}\right)=0$ and $\delta\left(y \otimes\left(x^{s}\right)_{*}\right)=(-1)^{s}\left(\begin{array}{c}m \\ s\end{array}\right)\left(x \otimes 1_{*}\right)^{m-s}$, where $\operatorname{deg} x \otimes 1_{*}=l$ and $\operatorname{deg}\left(y \otimes\left(x^{s}\right)_{*}\right)=l m-l s-1$. Then the rational model $m(e v)$ for the evaluation map $e v: \operatorname{aut}_{1}(M) \times M \rightarrow M$ is given by $m(e v)(x)=\left(x \otimes 1_{*}\right) \otimes 1+1 \otimes x$ and

$$
m(e v)(y)=\sum_{s=0}^{m-1}(-1)^{s}\left(y \otimes\left(x^{s}\right)_{*}\right) \otimes x^{s}+1 \otimes y .
$$




\section{A MINIMAL MODEL FOR $\operatorname{aut}_{1}(M)$}

Let $M$ be a space whose rational cohomology is isomorphic to an algebra of the form (1.1). Observe that $M$ is a formal space with $\operatorname{dim} H^{*}(M)<\infty$. Thus the construction in Section 2 of the HBS model for $\operatorname{aut}_{1}(M)$ is applicable. We here construct a relevant minimal model for $\operatorname{aut}_{1}(M)$ with the HBS model.

We first take a minimal model $(\wedge V, d)$ for $M$ for which $\wedge V=\wedge\left(x_{1}, \ldots, x_{q}, \rho_{1}, \ldots, \rho_{q}\right)$ and $d\left(\rho_{i}\right)=u_{i}$ for $i=1, \ldots, q$. Let $\left\{m_{j}\right\}_{j \in J}$ be a basis of $H^{*}(M)$ and $T$ the set $\{1, \ldots, q\} \times J$ and $\left\{m_{j}\right\}_{j \in J}$. Consider the HBS model $E / M_{u}$ for $\operatorname{aut}_{1}(M)$ mentioned in Section 2. It is readily seen that the model has the form

$$
E / M_{u}=\left(\wedge\left(\rho_{i} \otimes\left(m_{j}\right)_{*} ;(i, j) \in T_{1}, x_{i} \otimes\left(m_{j}\right)_{*} ;(i, j) \in T_{2}\right), \delta\right),
$$

where $T_{1}=\left\{(i, j) \in T \mid \operatorname{deg} \rho_{i} \otimes\left(m_{j}\right)_{*} \geq 1\right\}$ and $T_{2}=\left\{(i, j) \in T \mid \operatorname{deg} x_{i} \otimes\left(m_{j}\right)_{*} \geq\right.$ $1\}$. We define the linear part $\delta_{0}$ of the differential $\delta$ by $\delta(x)-\delta_{0}(x) \in\left(E / M_{u}\right)^{+}$. $\left(E / M_{u}\right)^{+}$. Then the part $\delta_{0}$ defines a linear map

$$
\delta_{0, \text { res }}: \mathbb{Q}\left\{\rho_{i} \otimes\left(m_{j}\right)_{*} ;(i, j) \in T_{1}\right\} \rightarrow \mathbb{Q}\left\{x_{i} \otimes\left(m_{j}\right)_{*} ;(i, j) \in T_{2}\right\},
$$

where $\mathbb{Q}\{U\}$ denotes the vector space generated by a set $U$.

As mentioned in Introduction, we are able to relate Halperin's conjecture with a property of the linear part $\delta_{0, \text { res }}$. Let $\operatorname{Der}_{\mathbb{Q}}\left(H^{*}(M)\right)_{-}$be the vector space of all derivations of negative degree of $H^{*}(M)$.

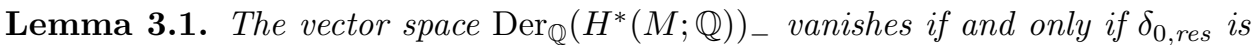
surjective.

Proof. It follows from [27, (2.6) Proposition (ii)] that the vector space of all derivations of negative degree of $H^{*}(M)$ is isomorphic to $\pi_{\text {even }}\left(\operatorname{aut}_{1}\left(M_{\mathbb{Q}}\right)\right)$. Moreover the result [ $\left[4\right.$, Theorem 15.11] enables us to conclude that $\operatorname{Coker} \delta_{0, \text { res }} \cong \pi_{\text {even }}\left(\operatorname{aut}_{1}\left(M_{\mathbb{Q}}\right)\right)$. This completes the proof.

In order to construct a minimal model for $E / M_{u}$, we arrange bases $\rho_{i} \otimes\left(m_{j}\right)_{*}$ and $x_{i} \otimes\left(m_{j}\right)_{*}$ as follows. Fix total orders on the sets $T_{1}$ and $T_{2}$, respectively. Using the orders, we define column vectors $\left(\delta_{0, \text { res }}\left(\rho_{i} \otimes\left(m_{j}\right)_{*}\right)\right)$ and $\left(x_{i} \otimes\left(m_{j}\right)_{*}\right)$.

Suppose that $\operatorname{Der}_{\mathbb{Q}}\left(H^{*}(M ; \mathbb{Q})\right)_{-}=0$. Then it follows from Lemma 3.1 that there exists a $(k, l)$-matrix $A$ with full rank such that

$$
A\left(\delta_{0, \mathrm{res}}\left(\rho_{i} \otimes\left(m_{j}\right)_{*}\right)\right)=\left(x_{i} \otimes\left(m_{j}\right)_{*}\right),
$$

where $k=\sharp T_{2}$ and $l=\sharp T_{1}$. Thus, changing the order on $T_{1}$ if necessary, we can find a regular $(k, k)$-matrix $B$ such that $B A=(E, *)$, where $E$ is the identity matrix. Put $B\left(x_{i} \otimes\left(m_{j}\right)_{*}\right)=\left(v_{l}\right)$. We choose the pair $(s, t) \in T_{1}$ which satisfies the condition that

$$
\sharp\left\{(i, j) \in T_{1} \mid(i, j) \leq(s, t)\right\}=k .
$$


We have

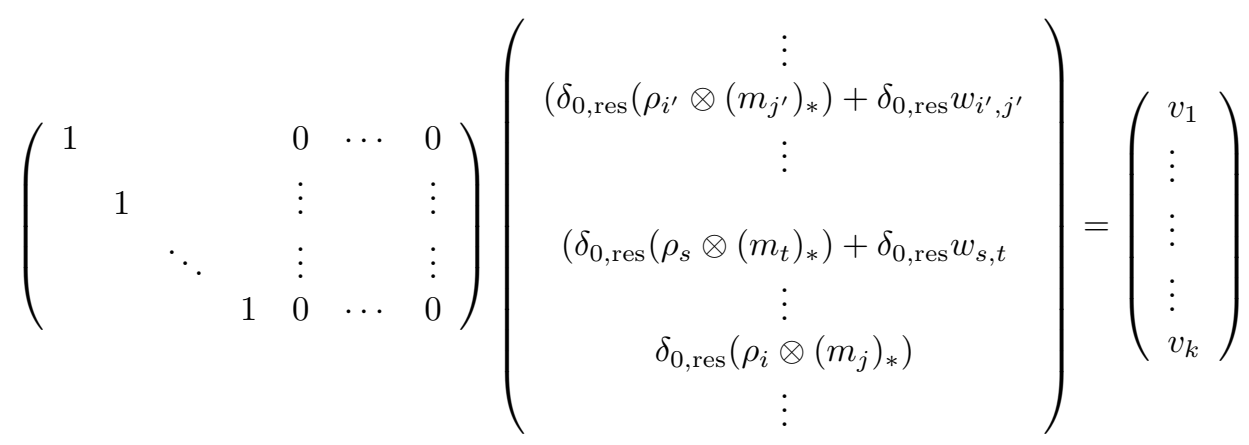

for which each $w_{i^{\prime}, j^{\prime}}$ is a linear combination of elements $\rho_{i} \otimes\left(m_{j}\right)_{*}$ with $(i, j)>$ $(s, t)$. Without loss of generality, we can assume that $(i, 0)>(s, t)$ for $i=1, \ldots, q$ because $\delta_{0, \text { res }}\left(\rho_{i} \otimes 1_{*}\right)=0$ for any $i$. We write $\left(b_{1}, \ldots, b_{k}\right)=\left(\cdots, \rho_{i^{\prime}} \otimes\left(m_{j^{\prime}}\right)_{*}+\right.$ $\left.w_{i^{\prime}, j^{\prime}}, \cdots, \rho_{s} \otimes\left(m_{t}\right)_{*}+w_{s, t}\right)$. Observe that $b_{1}, \ldots, b_{k}, \rho_{i} \otimes\left(m_{j}\right)_{*} ;(i, j)>(s, t)$ are linearly independent. Since $v_{1}, \ldots, v_{k}$ generate the image of $\delta_{0, \text { res }}$, it follows that for any $(i, j)$ greater than $(s, t)$,

$$
\delta_{0, \text { res }}\left(\rho_{i} \otimes\left(m_{j}\right)_{*}\right)-\delta_{0, \text { res }}\left(\sum_{l} \alpha_{i l} b_{l}\right)=0
$$

for some $\alpha_{i l} \in \mathbb{Q}$. Thus we have

$$
\left.E / M_{u} \cong \wedge\left(b_{1}, \ldots, b_{k}, \ldots, \rho_{i} \otimes\left(m_{j}\right)_{*}+\sum_{l} \alpha_{i l} b_{l}, \ldots, v_{1}, \ldots, v_{k}\right), \delta\right)
$$

for which $\delta b_{i} \equiv v_{i}$ and $\delta\left(\rho_{i} \otimes\left(m_{j}\right)_{*}+\sum_{l} \alpha_{i l} b_{l}\right) \equiv 0$ modulo decomposable elements. Put $S:=\left\{(i, j) \in T_{1} \mid(i, j)>(s, t)\right\}$. It is immediate that $S_{0} \subset S$. We then have a retraction

$$
r: E / M_{u} \rightarrow \wedge Z:=\left(\wedge\left(\rho_{i} \otimes\left(m_{j}\right)_{*} ;(i, j) \in S\right), 0\right)
$$

defined by $r\left(\left(\rho_{i} \otimes\left(m_{j}\right)_{*}\right)=0\right.$ if $(i, j) \notin S, r\left(v_{i}\right)=0$ and $r\left(\left(\rho_{i} \otimes\left(m_{j}\right)_{*}\right)=\rho_{i} \otimes\right.$ $\left(m_{j}\right)_{*}$ if $(i, j) \in S$. Since each term of the decomposable elements $\delta b_{i}-v_{i}$ and $\delta\left(\rho_{i} \otimes\left(m_{j}\right)_{*}+\sum_{l} \alpha_{i l} b_{l}\right)$ contain $v_{l}$ for some $l$ as a factor, we see that $\gamma$ is a welldefined DGA map.

The fact that $r$ is a quasi-isomorphism follows from the usual spectral sequence argument.

\section{An approximation to the Eilenberg-Moore spectral sequence}

In order to prove Theorem 1.1, we introduce a spectral sequence. Let $C_{*}(X)$ denote the normalized chain complex of a space $X$ with coefficients in the rational field. By definition, the total space $M_{\text {aut }_{1}(M)}$ of the universal $M$-fibration is regarded as the realization $\mid B_{*}\left(*\right.$ aut $\left._{1}(M), M\right) \mid$ of the geometric bar construction $B_{*}\left(*, \operatorname{aut}_{1}(M), M\right)$, which is a simplicial topological space with $B_{i}\left(*, \operatorname{aut}_{1}(M), M\right)=$ $* \times \operatorname{aut}_{1}(M)^{\times i} \times M$; see [24, Proposition 7.9]. The result [24, Theorem 13.9] allows us to obtain natural quasi-isomorphisms which connect with $C_{*}\left(\left|B\left(*, \operatorname{aut}_{1}(M), M\right)\right|\right)$ and the algebraic bar construction of the form $B\left(C_{*}(*), C_{*}\left(\operatorname{aut}_{1}(M)\right), C_{*}(M)\right)$ for which

$$
B\left(C_{*}(*), C_{*}\left(\operatorname{aut}_{1}(M)\right), C_{*}(M)\right)_{k}=\oplus_{i+j=k}\left(C_{*}(*) \otimes C_{*}\left(\operatorname{aut}_{1}(M)\right)^{\otimes i} \otimes C_{*}(M)\right)_{j} .
$$


Moreover the Eilenberg-Zilber map gives rise to a quasi-isomorphism from the bar complex to the total complex $\operatorname{Total}_{*}\left(B_{*}(*\right.$, aut $\left.(M), M)\right)$. Observe that

$$
\operatorname{Total} C_{*}\left(B_{*}\left(*, \operatorname{aut}_{1}(M), M\right)\right)_{k}=\oplus_{i+j=k} C_{j} B_{i}\left(*, \operatorname{aut}_{1}(M), M\right) .
$$

In consequence, by virtue of [4, Corollary 10.10], we have natural quasi-isomorphisms which connect $C^{*}\left(M_{\text {aut }_{1}(M)}\right)=C^{*}\left(\mid B_{*}(*\right.$, aut $\left.1(M), M) \mid\right)$ with the total complex of a double complex $\mathcal{B}=\left\{\mathcal{B}^{i, j}, d_{i}, \delta_{j}\right\}$ of the form

$$
\mathcal{B}^{i, j}=\left(A_{P L}\left(\operatorname{aut}_{1}(M)^{\times i} \times M\right)\right)^{j} .
$$

In particular, $d_{0}: \mathcal{B}^{0, *} \rightarrow \mathcal{B}^{1, *}$ is regarded as the map

$$
\left(p r_{2}\right)^{*}-e v^{*}: A_{P L}(M) \rightarrow A_{P L}\left(\operatorname{aut}_{1}(M) \times M\right),
$$

where the maps $p r_{2}$ and $e v$ from $\operatorname{aut}_{1}(M) \times M \rightarrow M$ to $M$ denote the second projection and the evaluation map, respectively.

We define a double complex $\mathcal{C}=\left\{\mathcal{C}^{i, j}, d_{i}, \delta_{j}\right\}$ by truncating the double complex $\left\{\mathcal{B}^{i, j}\right\}$ for $i \geq 2$; that is, $\mathcal{C}^{i, j}=\mathcal{B}^{i, j}$ for $i \leq 0,1$ and $\mathcal{C}^{i, j}=0$ for $i \geq 2$.

Let $\left\{E_{r}, \bar{d}_{r}\right\}$ be the Eilenberg-Moore spectral sequence converging to the rational cohomology $H^{*}\left(M_{\operatorname{aut}_{1}(M)}\right)$ with

$$
E_{2}^{*, *} \cong \operatorname{Cotor}_{H^{*}\left(\operatorname{aut}_{1}(M)\right)}^{* *}\left(\mathbb{Q}, H^{*}(M)\right)
$$

as an algebra. Observe that this spectral sequence is constructed with the double complex $\mathcal{B}$. The double complex $\mathcal{C}$ gives rise to a spectral sequence $\left\{\widetilde{E}_{r}, \widetilde{d}_{r}\right\}$ converging to $H^{*}(\operatorname{Total}(\mathcal{C}))$. Moreover, we see that the projection $q: \mathcal{B} \rightarrow \mathcal{C}$ induces the morphism of the spectral sequences

$$
\left\{q_{r}\right\}:\left\{E_{r}, d_{r}\right\} \rightarrow\left\{\widetilde{E}_{r}, \widetilde{d}_{r}\right\}
$$

and the morphism $\widehat{q}: H^{*}\left(M_{\text {aut }_{1}(M)}\right) \rightarrow H^{*}(\operatorname{Total}(\mathcal{C}))$ of algebras.

Lemma 4.1. For any $\alpha \in H^{*}\left(M_{\operatorname{aut}_{1}(M)}\right), \widehat{q}(\alpha)=0$ if and only if $\alpha \in F^{2} H^{*}$. Here $\left\{F^{p} H^{*}\right\}_{p \geq 0}$ denotes the filtration of $H^{*}\left(M_{\mathrm{aut}_{1}(M)}\right)$ associated with the spectral sequence $\left\{E_{r}, \bar{d}_{r}\right\}$.

Proof. By construction, we see that $q_{2}: E_{2}^{p, *} \rightarrow \widetilde{E}_{2}^{p, *}$ is bijective for $p=0$ and injective for $p=1$. Since the spectral sequence $\left\{\widetilde{E}_{r}, \widetilde{d}_{r}\right\}$ collapses at the $E_{1}$-term, it follows that the map $q_{r}^{p, *}$ for $3 \leq r \leq \infty$ and $p \leq 1$ is injective. This completes the proof.

Let $M$ be a space as in Theorem 1.1 and $E / M_{u}$ the HBS model for the monoid aut $_{1}(M)$ mentioned in Section 2. Since $M$ is formal, we can take a quasi-isomorphism $\eta:(\wedge V, d) \stackrel{\simeq}{\rightrightarrows} H^{*}(M ; \mathbb{Q})$. Moreover let $m(e v): \wedge V \rightarrow E / M_{u} \otimes \wedge V$ denote the model for the evaluation map $e v: \operatorname{aut}_{1}(M) \times M \rightarrow M$ described in Proposition 2.1. Recall the retraction $r: E / M_{u} \rightarrow \wedge Z$ mentioned in Section 3. We then have 
a commutative diagram

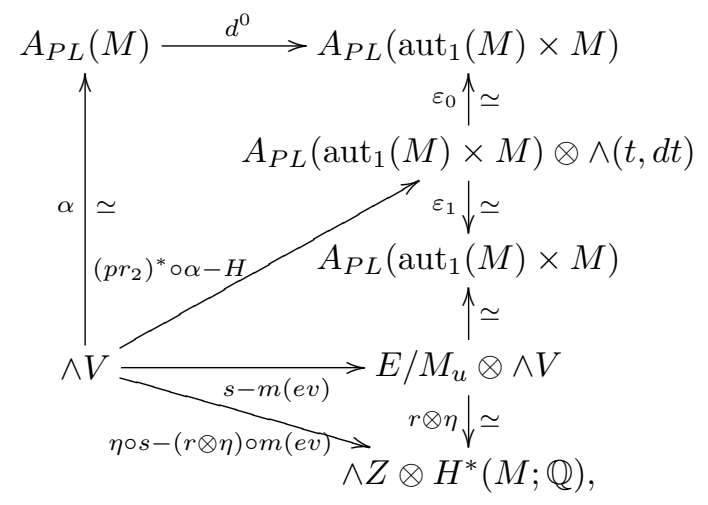

where $H: \wedge V \rightarrow A_{P L}\left(\operatorname{aut}_{1}(M) \times M\right) \otimes \wedge(t, d t)$ denotes the homotopy between the model $m(e v)$ for the evaluation map ev and the induced map $A_{P L}(e v)$ up to quasi-isomorphisms and $s$ stands for the inclusion into the second factor.

Let $\mathcal{D}$ be the double complex associated with the differential graded $\mathbb{Q}$-module map

$$
\eta \circ s-(r \otimes \eta) \circ m(e v):\left(\wedge V, d_{\wedge V}\right) \rightarrow \wedge Z \otimes H^{*}(M ; \mathbb{Q})
$$

Observe that $\mathcal{D}^{i, j}=0$ for $i \geq 2$. The usual spectral sequence argument allows us to conclude that $H^{*}($ total $\mathcal{C}) \cong H^{*}($ total $\mathcal{D})$ as a vector pace. By using this identification, we shall prove Theorem 1.1.

\section{Proof of Theorem 1.1}

We use the same notation as in Sections 3 and 4 throughout this section. Proof of Theorem 1.1. Recall from Section 3 the minimal model $\wedge Z$ for the monoid $\operatorname{aut}_{1}(M)$; that is,

$$
\wedge Z=\left(\wedge\left(\rho_{i} \otimes\left(m_{j}\right)_{*},(i, j) \in S\right), 0\right),
$$

where $\left\{\left(m_{j}\right)_{*}\right\}_{j \in J}$ denotes the dual basis of $H^{*}(M ; \mathbb{Q})$ and $\operatorname{deg} \rho_{i} \otimes\left(m_{j}\right)_{*}=\operatorname{deg} u_{i}-$ $1-\operatorname{deg} m_{j}$. We observe that $\operatorname{Der}_{\mathbb{Q}}\left(H^{*}(M ; \mathbb{Q})\right)_{-}=0$ by assumption. Thus we have

$$
H^{*}\left(\operatorname{aut}_{1}(M)\right) \cong \wedge\left(\rho_{i} \otimes\left(m_{j}\right)_{*},(i, j) \in S\right),
$$

This yields that, in the Leray-Serre spectral sequence for the universal fibration $\operatorname{aut}_{1}(M) \rightarrow \operatorname{Eaut}_{1}(M) \stackrel{\pi^{\prime}}{\rightarrow} \operatorname{Baut}_{1}(M)$, the element $\rho_{i} \otimes\left(m_{j}\right)_{*}$ is transgressive for any $(i, j) \in S$. In fact we have a commutative diagram

$$
\begin{aligned}
& \pi_{*}\left(\operatorname{aut}_{1}(M)\right)^{\sharp} \stackrel{\delta^{\sharp}}{\cong} \pi_{*+1}\left(E_{\operatorname{aut}_{1}(M)}, \operatorname{aut}_{1}(M)\right)^{\sharp} \underset{\pi_{*}^{\prime \sharp}}{\cong} \pi_{*+1}\left(\operatorname{Baut}_{1}(M)\right)^{\sharp} \\
& \uparrow_{h_{G}^{\sharp}} \quad \uparrow_{(-1)^{*+1} h^{\sharp}} \cong \uparrow_{(-1)^{*+1} h_{B G}^{\sharp}} \\
& H^{*}\left(\operatorname{aut}_{1}(M)\right) \stackrel{\delta}{\cong} H^{*+1}\left(E_{\operatorname{aut}_{1}(M)}, \operatorname{aut}_{1}(M)\right) \stackrel{\pi^{\prime *}}{\longleftarrow} H^{*+1}\left(\operatorname{Baut}_{1}(M)\right),
\end{aligned}
$$

where $h_{G}, h$ and $h_{B G}$ denote the duals to Hurewicz maps. Since $H^{*}\left(\operatorname{aut}_{1}(M)\right)$ and $H^{*}\left(\operatorname{Baut}_{1}(M)\right)$ are exterior algebra and a polynomial algebra, respectively, it follows that $h_{G}$ and $h_{B G}$ are isomorphisms on vector subspaces of indecomposable elements. Thus we see that the element $\rho_{i} \otimes\left(m_{j}\right)_{*}$ is transgressive because the map $\delta^{-1} \pi^{\prime *}$ is the transgression by definition. Thus the result [29, Section $\left.7(2.27)\right]$ implies that the element $\rho_{i} \otimes\left(m_{j}\right)_{*}$ is primitive for $(i, j) \in S$. 
Let $\left\{\widehat{E}_{r}, \widehat{d}_{r}\right\}$ be the Eilenberg-Moore spectral sequence converging to the cohomology $H^{*}\left(\operatorname{Baut}_{1}(M)\right)$ with $\widehat{E}_{2}^{*, *} \cong \operatorname{Cotor}_{H^{*}\left(\operatorname{aut}_{1}(M)\right)}^{* *}(\mathbb{Q}, \mathbb{Q})$. Since the element $\rho_{i} \otimes\left(m_{j}\right)_{*}$ is primitive for $1 \leq i \leq m$, it follows that

$$
\widehat{E}_{2}^{*, *}=\mathbb{Q}\left[\left[\rho_{i} \otimes\left(m_{j}\right)_{*}\right],(i, j) \in S\right],
$$

where bideg $\left[\rho_{i} \otimes\left(m_{j}\right)_{*}\right]=\left(1, \operatorname{deg} \rho_{i}-\operatorname{deg} m_{j}\right)$. This allows us to conclude that, as algebras,

$$
H^{*}\left(\operatorname{Baut}_{1}(M)\right) \cong \operatorname{Total}\left(\widehat{E}_{2}^{*, *}\right) \cong \mathbb{Q}\left[\left[\rho_{i} \otimes\left(m_{j}\right)_{*}\right],(i, j) \in S\right] .
$$

Recall the Eilenberg-Moore spectral sequence $\left\{E_{r}, d_{r}\right\}$ converging to the cohomology ring $H^{*}\left(M_{\text {aut }_{1}(M)}\right)$. We see that

$$
\begin{aligned}
E_{2}^{*, *} & \cong \operatorname{Cotor}_{H^{*}\left(\operatorname{aut}_{1}(M)\right)}^{*}\left(\mathbb{Q}, H^{*}(M)\right) \\
& \cong \mathbb{Q}\left[\left[\rho_{i} \otimes\left(m_{j}\right)_{*}\right],(i, j) \in S\right] \otimes H^{*}(M)
\end{aligned}
$$

as bigraded algebras. For dimensional reasons, we see that the spectral sequence $\left\{E_{r}, d_{r}\right\}$ collapses at the $E_{2}$-term. Let $M \stackrel{\iota}{\rightarrow} M_{\text {aut }_{1}(M)} \stackrel{\pi}{\rightarrow} B$ aut $_{1}(M)$ be the universal $M$-fibration. The naturality of the spectral sequence enables us to deduce that $\pi^{*}\left(\left[\rho_{i} \otimes\left(m_{j}\right)_{*}\right]\right)=\left[\rho_{i} \otimes\left(m_{j}\right)_{*}\right]$ in $H^{*}\left(M_{\text {aut }_{1}(M)}\right)$. In the total complex of the double complex $\mathcal{D}$, we have

$$
\begin{aligned}
\left(d_{\wedge V} \pm(\eta s-(r \otimes \eta) \circ m(e v))\right) \rho_{i} & =d_{\wedge V}\left(\rho_{i}\right)+(-1)^{\operatorname{deg} \rho_{i}}(\eta s-(r \otimes \eta) \circ m(e v))\left(\rho_{i}\right) \\
& =\left([] u_{i}\right)+\sum_{(i, j) \in S}(-1)^{\operatorname{deg} m_{j} / 2}\left[\rho_{i} \otimes\left(m_{j}\right)_{*}\right] \otimes m_{j} .
\end{aligned}
$$

This implies that $\widehat{q}\left(\left([] u_{i}\right)+\sum_{(i, j) \in S}(-1)^{\operatorname{deg} m_{j} / 2}\left[\rho_{i} \otimes\left(m_{j}\right)_{*}\right] \otimes\left([] m_{j}\right)\right)=0$ in $H^{*}(\operatorname{Total}(\mathcal{C}))$. Therefore it follows from Lemma 4.1 that

$$
\left([] u_{i}\right) \equiv \sum_{(i, j) \in S} \nu_{(i, j)} \otimes m_{j}
$$

modulo the ideal generated by $\pi^{*}\left(H^{*}\left(B \operatorname{Baut}_{1}(M)\right)^{+} \cdot H^{*}\left(B \operatorname{aut}_{1}(M)\right)^{+}\right)$in $H^{*}\left(M_{\text {aut }_{1}(M)}\right)$, where $\nu_{(i, j)}=(-1)^{\left(\operatorname{deg} m_{j} / 2\right)+1}\left[\rho_{i} \otimes\left(m_{j}\right)_{*}\right]$.

The latter half of Theorem 1.1 follows from the equalities

$$
\begin{aligned}
\sharp\left\{\nu_{s} \mid \operatorname{deg} \nu_{s}=2 k, s \in S\right\}= & \operatorname{dim} \pi_{2 k}\left(\operatorname{Baut}_{1}\left(M_{\mathbb{Q}}\right)\right) \\
= & \operatorname{dim} \pi_{2 k-1}\left(\operatorname{aut}_{1}\left(M_{\mathbb{Q}}\right)\right) \\
= & \sharp\left\{\rho_{i} \otimes\left(m_{j}\right)_{*} \mid \operatorname{deg} \rho_{i} \otimes\left(m_{j}\right)_{*}=2 k-1,(i, j) \in S\right\} \\
= & \operatorname{rank} \oplus_{a-b=2 k-1} \pi_{a}(M) \otimes H^{b}(X ; \mathbb{Q}) \\
& -\operatorname{rank} \oplus_{a-b=2 k} \pi_{a}(M) \otimes H^{b}(X ; \mathbb{Q}) .
\end{aligned}
$$

This completes the proof.

Proof of Proposition 1.3. As is well-known, Halperin's conjecture is affirmatively solved for the given space $M$. Then Theorem 1.1 is applicable. We choose a basis of the form $\left\{a^{i}\right\}_{0 \leq i \leq m}$ of $H^{*}(M ; \mathbb{Q})$. There is no element $w$ in $\pi^{*}\left(H^{*}\left(B \operatorname{aut}_{1}(M) ; \mathbb{Q}\right)^{+}\right.$. $\left.H^{*}\left(\operatorname{Baut}_{1}(M) ; \mathbb{Q}\right)^{+}\right)$such that $\operatorname{deg} a^{m+1}=\operatorname{deg} w a^{m}$. This yields that each term of $D_{1}$ does not have $a^{m}$ as a factor. Replace the indecomposable element $\nu_{(1, j)}$ in Theorem 1.1 with $\mu_{j}=\nu_{(1, j)}+\xi_{j}$ for any $j \geq 2$, where $\xi_{j}$ is the coefficient of $a^{m-j+1}$ in $D_{1}$. We have the result. 
Remark 5.1. Let $M$ be a space as in Theorem 1.1. Recall the subset $S$ of $\{1, \ldots, q\} \times J$ mentioned in the construction of the minimal model for $\operatorname{aut}_{1}(M)$ in Section 3. Let $\rho:\{1, \ldots, q\} \times J \rightarrow J$ be the projection. Suppose that $\operatorname{deg} u_{i} \neq \operatorname{deg} w m_{j}$ for any $(i, j) \in\{1, \ldots, q\} \times(J \backslash \rho(S))$ and $w \in \pi^{*}\left(H^{*}\left(\operatorname{Baut}_{1}(M) ; \mathbb{Q}\right)^{+} \cdot H^{*}\left(\operatorname{Baut}_{1}(M) ; \mathbb{Q}\right)^{+}\right)$. As in the proof of Proposition 1.3, we can arrange the indecomposable elements $\nu_{s}$ so that each $D_{i}$ in Theorem 1.1 is trivial.

\section{6. $\mu$-CLASSES}

In order to define $\mu$-classes due to Kedra and McDuff, we first recall the coupling class. Let $(M, a)$ be a c-symplectic space with formal dimension $k=2 m$. Consider the Leray-Serre spectral sequence $\left\{E_{r}, d_{r}\right\}$ for a fibration $M \stackrel{i}{\rightarrow} E \stackrel{\pi}{\rightarrow} B$ for which $\pi_{1}(B)$ act trivially on $H^{k}(M)=\mathbb{Q}$. Let $\left\{F^{p} H^{*}\right\}_{p \geq 0}$ denote the filtration of $\left\{E_{r}, d_{r}\right\}$. Then the integration along the fibre (the cohomology push forward) $\pi !: H^{p+k}(E) \rightarrow H^{p}(B)$ is defined by the composite

$$
H^{p+k}(E)=F^{0} H^{p+k}=F^{p} H^{p+k} \rightarrow E_{\infty}^{p, q} \longmapsto \cdot \longmapsto E_{2}^{p, q} \cong H^{p}\left(B ; H^{k}(M)\right) \cong H^{p}(B) .
$$

Let $\mathcal{G}$ denote the group $\mathcal{H}_{a}$ or the monoid $\operatorname{aut}_{1}(M)$. Let $M \stackrel{\iota}{\rightarrow} M_{\mathcal{G}} \stackrel{\pi}{\rightarrow} B \mathcal{G}$ be the universal $M$-fibration; see [24, Proposition 7.9]. Proposition 6.1 below follows from the proofs of [12, Proposition 2.4.2] and [13, Proposition 3.1].

Proposition 6.1. Suppose that $H^{1}(M)=0$, then the element $a \in H^{2}(M)$ is extendable to an element $\bar{a} \in H^{2}\left(M_{\mathcal{G}}\right)$. Moreover, there exists a unique element $\widetilde{a} \in H^{2}\left(M_{\mathcal{G}}\right)$ that restricts to $a \in H^{2}(M)$ and such that $\pi !\left(\widetilde{a}^{m+1}\right)=0$. In fact the element $\widetilde{a}$ has the form

$$
\widetilde{a}=\bar{a}-\frac{1}{n+1} \pi^{*} \pi !\left(\bar{a}^{m+1}\right) .
$$

The class $\widetilde{\omega}$ in Proposition 6.1 is called the coupling class.

Definition 6.2. [13, Section 3.1] [12, Section 2.4] [30] We define $\mu_{k} \in H^{2 k}(B \mathcal{G})$, which is called $k$ th $\mu$-class, by

$$
\mu_{k}:=\pi !\left(\widetilde{a}^{m+k}\right)
$$

where $\widetilde{a}$ is the coupling class.

Proof of Proposition 1.4. We use the same notation as in the previous sections. It follows from Lemma 4.1 that

$$
([] a)^{m+1} \equiv \sum_{i=1}^{m}(-1)^{m-i+1}\left[y \otimes\left(a^{m-i}\right)_{*}\right] \otimes([] a)^{m-i}
$$

modulo the ideal generated by $\pi^{*}\left(H^{*}\left(\operatorname{Baut}_{1}(M)\right)^{+} \cdot H^{*}\left(\operatorname{Baut}_{1}(M)\right)^{+}\right)$in $H^{*}\left(M_{\text {aut }_{1}(M)}\right)$. Since $H^{2}\left(\operatorname{Baut}_{1}(M)\right)=0$, the definition of the integration $\pi$ ! enables us to deduce that $\pi !\left(([] a)^{m+1}\right)=0$. We can choose the element $([] a)$ as the coupling class $\widetilde{a}$ mentioned in Proposition 6.1. By definition, for $2 \leq k \leq m+1$, we see that

$$
\begin{aligned}
\mu_{k} & =\pi !\left(\widetilde{a}^{m+k}\right)=\pi !\left(\widetilde{a}^{m+1} \cdot \widetilde{a}^{k-1}\right) \\
& =\pi !\left(\left(\sum_{i=1}^{m}(-1)^{m-i+1}\left[y \otimes\left(a^{m-i}\right)_{*}\right] \widetilde{a}^{m-i}\right) \cdot \widetilde{a}^{k-1}\right) \\
& =\pi !\left(\cdots+(-1)^{m-k}\left[y \otimes\left(a^{m-k+1}\right)_{*}\right] \widetilde{a}^{m}+\cdots\right) \\
& =(-1)^{m-k}\left[y \otimes\left(a^{m-k+1}\right)_{*}\right]
\end{aligned}
$$


modulo decomposable elements. We have the result.

Remark 6.3. Let $M$ be a c-symplectic space of the form $\left(\mathbb{C} P^{m} \times \mathbb{C} P^{n}, a_{1}+a_{2}\right)$. Then it follows that $\mu$-classes do not generate the whole algebra $H^{*}\left(\operatorname{Baut}_{1}(M)\right)$. To see this, we choose minimal models $\left(\wedge\left(y_{1}, a_{1}\right), d y_{1}=a_{1}^{m+1}\right)$ and $\left(\wedge\left(y_{2}, a_{2}\right), d y_{2}=a_{1}^{n+1}\right)$ for the projective spaces $\mathbb{C} P^{m}$ and $\mathbb{C} P^{n}$, respectively. Suppose that $m \geq n$. Then the same argument as in [11, Example 3.6] allows us to conclude that $\operatorname{aut}_{1}(M)$ admits a minimal model of the form

$$
\begin{gathered}
\wedge\left(y_{1} \otimes 1_{*}, y_{1} \otimes\left(a_{1}\right)_{*}, \ldots, y_{1} \otimes\left(a_{1}^{m-1}\right)_{*}, y_{2} \otimes 1_{*}, y_{2} \otimes\left(a_{2}\right)_{*}, \ldots, y_{2} \otimes\left(a_{2}^{n-1}\right)_{*},\right. \\
\left.y_{1} \otimes\left(a_{1}^{k_{1}} a_{2}^{l_{1}}\right)_{*}, y_{2} \otimes\left(a_{1}^{k_{2}} a_{2}^{l_{2}}\right)_{*} ; 1 \leq k_{1}+l_{1} \leq m, 0<l_{1} \leq n, 1 \leq k_{2}+l_{2} \leq n, k_{2} \neq 0\right)
\end{gathered}
$$

with the trivial differential. This yields that $H^{2}\left(\operatorname{Baut}_{1}(M)\right)$ is isomorphic to

$$
\mathbb{Q}\left\{\left[y_{1} \otimes\left(a_{1}^{k_{1}} a_{2}^{l_{1}}\right)_{*}\right],\left[y_{2} \otimes\left(a_{1}^{k_{2}} a_{2}^{l_{2}}\right)_{*}\right] ; k_{1}+l_{1}=m, 0<l_{1} \leq n, k_{2}+l_{2}=n, k_{2} \neq 0\right\}
$$

as a vector space. Thus any of $\mu$-classes does not detect an element in $H^{2}\left(\operatorname{Baut}_{1}(M)\right)$ since the degrees of the $\mu$-classes are greater than 4 .

We also determine the $H^{*}\left(\operatorname{Baut}_{1}(M) ; \mathbb{Q}\right)$-algebra structure of $H^{*}\left(M_{\operatorname{aut}_{1}(M)} ; \mathbb{Q}\right)$. It follows from Remark 5.1 that

$$
\begin{aligned}
& H^{*}\left(\operatorname{Baut}_{1}(M) ; \mathbb{Q}\right) \cong \\
& \mathbb{Q}\left[\begin{array}{l}
\nu\left(k_{1}^{\prime}, 0\right), \nu\left(k_{1}, l_{1}\right) \quad ; \quad 0 \leq k_{1}^{\prime} \leq m-1,1 \leq k_{1}+l_{1} \leq m, 1 \leq l_{1} \leq n \\
\eta\left(0, l_{1}^{\prime}\right), \eta\left(k_{2}, l_{2}\right)
\end{array}\right]
\end{aligned}
$$

and, as an $H^{*}\left(\operatorname{Baut}_{1}(M) ; \mathbb{Q}\right)$-algebra,

$$
H^{*}\left(M_{\text {aut }_{1}(M)} ; \mathbb{Q}\right) \cong H^{*}\left(\operatorname{Baut}_{1}(M) ; \mathbb{Q}\right) \otimes \mathbb{Q}\left[a_{1}, a_{2}\right] /\left(\rho_{1}, \rho_{2}\right),
$$

where

$$
\begin{aligned}
\rho_{1} & =a_{1}^{m+1}-\sum_{k_{1}^{\prime}=0}^{m-1} \nu\left(k_{1}^{\prime}, 0\right) a_{1}^{k_{1}^{\prime}}-\sum_{1 \leq k_{1}+l_{1} \leq m, 1 \leq l_{1} \leq n} \nu\left(k_{1}, l_{1}\right) a_{1}^{k_{1}} a_{2}^{l_{1}} \\
\rho_{1} & =a_{2}^{n+1}-\sum_{l_{1}^{\prime}=0}^{n-1} \eta\left(0, l_{1}^{\prime}\right) a_{2}^{l_{1}^{\prime}}-\sum_{1 \leq k_{2}+l_{2} \leq n, k_{2} \neq 0} \eta\left(k_{2}, l_{2}\right) a_{1}^{k_{2}} a_{2}^{l_{2}},
\end{aligned}
$$

$\operatorname{deg} \nu\left(k_{1}^{\prime}, 0\right)=2(m+1)-2 k_{1}^{\prime}, \operatorname{deg} \nu\left(k_{1}, l_{1}\right)=2(m+1)-2\left(k_{1}+l_{1}\right), \operatorname{deg} \eta\left(0, l_{1}^{\prime}\right)=$ $2(n+1)-2 l_{1}^{\prime}$ and $\operatorname{deg} \eta\left(k_{2}, l_{2}\right)=2(n+1)-2\left(k_{2}+l_{2}\right)$.

Proof of Proposition 1.5. We can take a Sullivan model $(\wedge V, d)$ for $M$ such that $\wedge V=\wedge\left(\chi, p_{1}^{\prime}, \ldots, p_{m-1}^{\prime}, \tau_{2}, \tau_{4}, \ldots, \tau_{2 m}\right)$ and $d\left(\tau_{2 i}\right)=(-1)^{i}\left(\chi^{2} p_{i-1}^{\prime}+p_{i}^{\prime}\right)$ for $1 \leq i \leq$ $m$. In view of the rational model for $\lambda_{G, M}: S O(2 m+1) \rightarrow$ aut $_{1}(M)$ mentioned in [17, Theorem 3.3], we have $\left(\lambda_{S O(2 m+1), M}\right)^{*}\left(\tau_{2 m} \otimes\left(\chi^{2 l}\right)_{*}\right)=\tau_{2(m-l)}$ in the cohomology; see also [17, Section 8 (1)]. Thus the naturality of the Eilenberg-Moore spectral sequence allows us to deduce that $\left(B \lambda_{S O(2 m+1), M}\right)^{*}\left(\left[\tau_{2 m} \otimes\left(\chi^{2 l}\right)_{*}\right]\right)=\left[\tau_{2(m-l)}\right]$. The description of the $\mu$-classes in the proof of Theorem 1.4 yields that

$$
\left(B \lambda_{S O(2 m+1), M}\right)^{*}\left(\mu_{2(m-l)}\right) \equiv\left(B \lambda_{S O(2 m+1), M}\right)^{*}\left(\left[\tau_{2 m} \otimes\left(\chi^{2 l}\right)_{*}\right]\right)
$$

modulo decomposable elements. Let $\sigma^{*}: H^{*}(B S O(2 m+1)) \rightarrow H^{*-1}(S O(2 m+1))$ be the cohomology suspension. Without loss of generality, we can assume that $\sigma^{*}\left(p_{m-l}\right)=\tau_{2(m-l)}$; see [4, Proposition 15.13]. By virtue of [7, Corollary 3.12], we have $\sigma^{*}\left(\left[\tau_{2(m-l)}\right]\right)=\tau_{2(m-l)}$. In our case, the cohomology suspension $\sigma^{*}$ is 
injective on the vector subspace of indecomposable elements. This implies that $\left[\tau_{2(m-l)}\right]=p_{m-l}$. We have the result.

Acknowledgments. The author thanks Toshihiro Yamaguchi for several helpful conversations during the early stages of this work.

\section{REFERENCES}

[1] E. H. Brown Jr and R. H. Szczarba, On the rational homotopy type of function spaces, Trans. Amer. Math. Soc. 349(1997), 4931-4951.

[2] U. Buijs and A. Murillo, Basic constructions in rational homotopy theory of function spaces, Ann. Inst. Fourier (Grenoble) 56(2006), 815-838.

[3] U. Buijs and A. Murillo, The rational homotopy Lie algebra of function spaces, Comment. Math. Helv. 83(2008), 723-739.

[4] Y. Félix, S. Halperin and J. -C. Thomas, Rational Homotopy Theory, Graduate Texts in Mathematics 205, Springer-Verlag.

[5] Y. Félix and J. -C. Thomas, The monoid of self-homotopy equivalences of some homogeneous spaces, Exposiotiones Math. 12(1994), 305-322.

[6] J. -B. Gatsinzi, On the genus of elliptic fibrations, Proc. Amer. Math. Soc. 132(2003), 597606.

[7] V. K. A. M. Gugenheim and J. P. May, On the theory and applications of differential torsion products, Mem. Amer. Math. Soc. 142, 1974.

[8] A. Haefliger, Rational homotopy of space of sections of a nilpotent bundle, Trans. Amer. Math. Soc. 273(1982), 609-620.

[9] S. Halperin, Finiteness in the minimal models of Sullivan, Trans. Amer. Math. Soc. 230(1977), 173-199.

[10] V. Hauschild, Deformations and the rational homotopy of the monoid of fibre homotopy equivalences, Illinois Journal of Math. 37(1993), 537-560.

[11] Y. Hirato, K. Kuribayashi and N. Oda, A function space model approach to the rational evaluation subgroups, Math. Z. 258(2008), 521-555.

[12] T. Januszkiewicz and J. Kedra, Characteristic classes of smooth fibrations, preprint (2002) arXiv:math/0209288v1.

[13] J. Kedra and D. McDuff, Homotopy properties of Hamiltonian group actions, Geometry \& Topology, 9(2005), 121-162.

[14] K. Kuribayashi, On the mod $p$ cohomology of the spaces of free loops on the Grassmann and Stiefel manifolds, J. Math. Soc. Japan 43(1991), 331-346.

[15] K. Kuribayashi, Module derivations and the adjoint acton of finite loop space, J. Math. Kyoto Univ. 39 (1999) 67-85.

[16] K. Kuribayashi, A rational model for the evaluation map, Georgian Mathematical Journal 13(2006), 127-141.

[17] K. Kuribayashi, Rational visibility of a Lie group in the monoid of selfhomotopy equivalences of a homogeneous space, preprint (2008), submitted, http://marine.shinshu-u.ac.jp/kuri/dvi/visibility-neo3.pdf.

[18] F. Lalonde and D. McDuff, Symplectic structures on fibre bundles, Topology, 42(2003), 309347.

[19] G. Lupton, Variations on a conjecture of Halperin, Homotopy and geometry (Warsaw, 1997), 115-135, Banach Center Publ., 45, Polish Acad. Sci., Warsaw, 1998.

[20] G. Lupton, Note on a conjecture of Stephen Halperin, Lecture Notes in Math., vol. 1440, Springer-Verlag (1990), 148-163.

[21] G. Lupton and J. Oprea, Cohomologically symplectic space: Toral actions and the Gottlieb group, Trans. Amer. Math. Soc. 347(1995), 261-288.

[22] M. Markl, Towards one conjecture on collapsing of the Serre spectral sequence, Rend. Circ. Mat. Palermo (2) Suppl. 22(1990), 151-159.

[23] H. Matsumura, Commutative ring theory, Cambridge Studies in Advanced Mathematics 8, Cambridge University Press, Cambridge, 1986.

[24] J.P.May, Classifying spaces and fibrations, Mem. Amer. Math. Soc. 155, 1975.

[25] J.P.May, Fiberwise localization and completion, Trans. Amer. Math. Soc. 258(1980), 127-146. 
[26] D. McDuff and D. Salamon, Introduction to Symplectic Topology, Oxford Mathematical Monographs, Clarendon Press, Oxford, 1995.

[27] W. Meier, Rational universal fibrations and flag manifolds. Math. Ann. 258(1982), 329-340.

[28] W. Meier, Some topological properties of Kähler manifolds and homogeneous spaces, Math. Z. 183(1983), 473-481.

[29] M. Mimura and H. Toda, Topology of Lie groups. II. Translations of Mathematical Monographs, 91. American Mathematical Society, Providence, RI, 1991.

[30] A. G. Reznikov, Characteristic classes in symplectic topology, Selecta Math. 3(1997), 601642 .

[31] H. Shiga ans M. Tezuka, Rational fibrations, homogeneous spaces with positive Euler characteristics and Jacobians, Ann. Inst. Fourier (Grenoble) 37(1987), 81-106.

[32] L. Smith, On the characteristic zero cohomology of the free loop space, Amer. J. Math. 103(1981), 887-910.

[33] S. B. Smith, The rational homotopy Lie algebra of classifying spaces for formal two-stage spaces, J. Pure Appl. Algebra 160(2001), 333-343.

[34] D. Tanré, Homotopie rationnelle: Modèles de Chen, Quillen, Sullivan, Lecture Notes in Math., no. 1025, Springer-Verlag, Berlin, 1983.

[35] J. -C. Thomas, Rational homotopy of Serre fibrations, Ann. Inst. Fourier (Grenoble) 31(1981), 71-90. 